\title{
Agent and Mobile Tools for Telehomecare in Developing Countries: An Architecture Approach
}

\author{
http://dx.doi.org/10.3991/ijim.v9i2.4245 \\ Karim Zarour \\ Lire laboratory, University Constantine 2, Algeria
}

\begin{abstract}
Nowadays, in the networked health, mobile tools are increasingly important, to provide care, educate patients or to transmit remote information. Telehomecare evolves constantly. It can aid disabled persons, increase patient compliance, enhance caregivers' effectiveness, and connect socially isolated individuals to their care providers. In this perspective, the agent paradigm proposes interesting concepts for the development of telehomecare information systems, such as the control autonomy, decentralization and coordination. This paper describes an architecture of telehomecare based on agents and mobile tools.
\end{abstract}

Index Terms-Telehomecare; Inter-Agent Communication; Electronic Medical Record; Information and Communication Technologies;

\section{INTRODUCTION}

Information and Communication Technologies (ICT) progress rapidly. The mobile tools have a large impact on the social life of citizens today [1]. Telemedicine in homecare, or telehomecare (THC), uses modern technologies to enable the communication and the transfer of information between the health care provider at the clinical site and the patient at his/her home. In THC, the patients conduct their interactions with their health care providers in their own homes [2]. Telehomecare is an emerging field that will benefit homecare providers and their patients. It does not replace in home care visits [3], but is used primarily to augment current services. Most countries are now facing an urgent requirement to provide appropriate retired home environment solutions for patients and allow them to stay at their own homes. Partial solutions for specific case studies of THC system are described in [4].

According to [5], most developed countries are facing important overall problems regarding healthcare services, such as: (i) increased demand of healthcare due to an increased number of elderly and changed life styles leading to an increase in chronic diseases (ii) demand for increased accessibility of care outside hospitals, moving health services into the patient's own homes (iii) need for increased efficiency, individualization and equity of quality-oriented healthcare with limited financial resources (iv) difficulties of recruiting and retaining personnel in the healthcare services in general and in home and elderly care in particular.

At the same time, there are several problems in homecare service in Algeria, such as: (i) current solutions based on manual note taking are slow (ii) obstacle to real- time data access (iii) medical staff is not up to date on the state of the patient (that curbs the ability of clinical diagnostics and monitoring) (iv) slow and laborious process of homecare (v) time consuming (vi) traditional data storage mode is not conducive to the sharing of resources. Therefore, there cannot be information sharing among all the stakeholders.

Telehomecare uses ICT to deliver patient care to the home [2];[5]. It is an emerging field that will benefit home care providers and their patients. It does not replace in home care visits, but is used primarily to augment current services. Examples of THC are: monitoring a patient's wound care progress, monitoring a newly diagnosed Diabetic patient's blood sugars, monitoring a chronic $\mathrm{CHF}$ patient for fluid overload with recording of daily weights. The goal is to inspire individuals to manage their own health at home [6];[7]. According to the literature, several definitions are attributed to the THC.

In the work of [8], THC is the use of technology to deliver patient care in the home or place of residence, providing patient-provider contact without either having to travel. In our context, we take consideration this definition. According to [9], THC is a specialisation of TM where the patient with or without support from family or local care do herself manage in monitoring and treatment under electronic observation from qualified clinical staff. Telehomecare is a subfield within telehealth. It involves the delivery of healthcare services to patients at home through the use of telecommunications technologies, which enable the interaction of voice, video, and health-related data explain [10]. The author of [11] uses the concept of home based eHealth to include both THC and smart homes.

Telehomecare is successful for certain groups of patients [12]:

- Patients requiring more than 2 home visits/week

- Chronic conditions

- Palliative conditions

Generally, users who can benefit from THC are [13]:

- People living alone who are unable to seek help in emergencies (unconsciousness, falls, strokes, myocardial infarction, etc.).

- Elderly or disabled people who suffer from cognitive (e.g. Alzheimer) and/or physical (visual, hearing, mobility, etc.) impairment.

- People who need help in daily life to perform personal care activities (e.g. toileting) and 
instrumental activities (cooking healthy meals, dealing with medication, and doing laundry).

- People living in rural and remote communities or in urban communities with inadequate health service provision.

- People who suffer from chronic disease, and who need continuous monitoring (e.g. diabetes)

- People involved in telehealth care undertaking health care at a distance or telemedicine, with physicians practising 'virtual visits'.

Telehomecare in developing countries [14] and particularly in Algeria is still facing many challenges especially in rural areas such as:

- Unavailability of the required ICT infrastructure for THC (e.g. internet connection)

- High cost of THC solutions.

- Lack of patients' awareness and acceptance of receiving healthcare services via THC networks and applications.

- Lack of uniform standards for data-sharing: Unified format and content of data storage are the basis for communication between systems. Information classification and data standards are not strictly uniform.

- $\quad$ Security, safety and privacy of data exchange.

In this paper, we propose a global architecture for THC, adapted to the concrete needs of the homecare in Algeria. This architecture allows the sharing, the fast and supple exchange of information, and opens new perspectives of flexibility.

The remainder of this paper is organised as follows. The architecture of the proposed system is detailed in Section 2 followed by mobile tools in section 3 . The prototype implementation is described in section 4 and related work of THC is dedicated in section 5. Finally, a conclusion with a view to the future is given in Section 6 .

\section{THE PROPOSED ARCHITECTURE}

The proposed architecture supports THC (see figure 1). This architecture is based on agents and mobile tools. Each actor (doctor, nurse, etc.) of Radiology Information Systems (RIS), Laboratory Information Systems, etc. is assisted by a software agent named User Agent (UA). The behavior of the agent is more organizational in the sense that it knows how to take into account the other members of homecare organization and to update its organizational knowledge. This architecture aims to facilitate the interoperability, the accessibility to information, and it supplies to homecare actors the same functionalities as if they belong all to a single structure (the same place). Our architecture is independent from any application in the field of patient's homecare (chronic diseases, old persons, etc.). Indeed, the main aims of our architecture to summarize in three points: (1) reducing delays in the management of patient (2) improve the efficiency of the THC team (3) and therefore, improve the service provided to patients and their satisfaction. A Virtual Private network (VPN) is established between all Information systems of the hospital, patient home and the data centre using wireless network links between them.
This is achieved by sourcing the services from an Internet Service Provider (ISP).

The measured data are transmitted via wireless connection; the information can be received via smart devices and uploaded in XML format through Internet. After, the system receives, processes and saves the data to the corresponding database location.

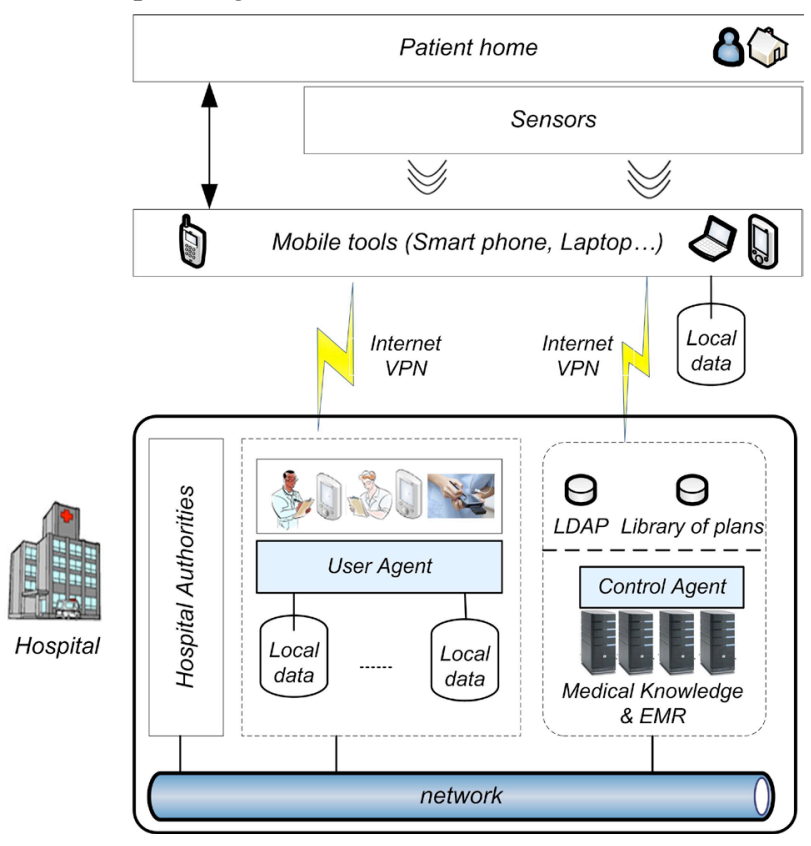

Figure 1. The overall information system architecture

\section{A. User agent (UA)}

It is a hybrid agent. It assists and helps an actor (doctor, nurse, etc.) that participates in the taking care of patients. Every user agent in the system can communicate with another one without passing by an intermediary. Its components are depicted in figure 2 :

User interface. It allows interaction between UA_and human agent. This is an interface of assistance for the human agent. This last one has a decision maker's role.

Coordination module. This module takes into consideration all of the overall process of coordination. It takes as input parameters a set of goals (the interpreted results of messages by the user interface and the communication module) and produces a plan which satisfies these purposes. The coordination module consists of a sub module of planning for the orientation and the organization of the local tasks and a sub module of negotiation for task allocation and the resolution of conflicts. For more details see our work in [3].

Individual knowledge module. An agent must have the ability to represent knowledge i.e. to remember and reason above. Thus, the individual knowledge base represents all information and knowledge about the agent himself: its abilities and competencies, the state and the charge of the current task. Accordingly, each UA of system keeps any information relative to its interventions, activities, etc. Example: If $\mathrm{X}$ has a body temperature $>40$ $\mathrm{X}$ has pressure 16-6 and if $\mathrm{X}$ has blood sugar level 3.8 ... then hospitalisation.

Homecare-knowledge module. It contains the 
information concerning the defined organizational and operational rules in a homecare. What allows having an organizational interoperability between the various partner agents involved in the system. It includes a list of all partner agents. This is knowledge that represents the know-how on the homecare for the good progress of the reasoning process and a coherent behaviour. Consequently, this knowledge base allows achieving the cooperation process and the management of interactions with the other UA. This module also contains information on rights and commitments for the homecare.

Communication module. This module manages the interaction between an UA and the outside world such as other agents (acquaintances). It contains all processes of taking charge of messages from other UA. This module is responsible for all functionalities of sending and receiving messages.

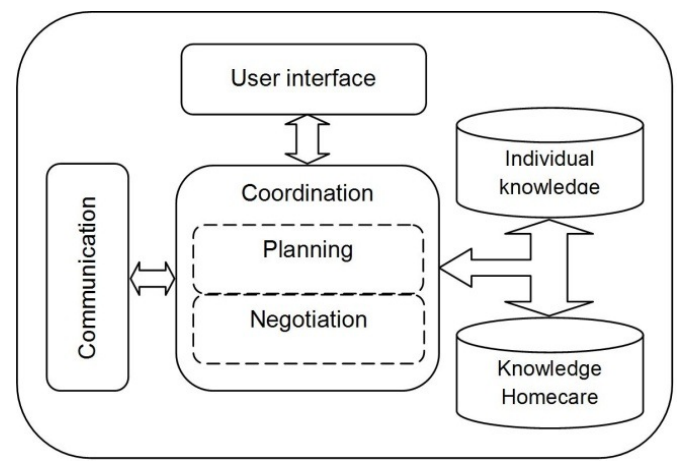

Figure 2. The structure of the user agent

\section{B. Local data}

Each database is designed to satisfy local needs and not necessarily to interact with other local databases. Indeed, the actors of THC and patients are very attached to their personal notes for their own use. But it is essential to share this information with other stakeholders.

\section{Hospital authorities}

They act on behalf of the hospital. These authorities have a number of powers: recommendation, decision, regulation and sanction. They also ensure some form of control over different medical staff:

LDAP (Lightweight Directory Access Protocol) directory. It is used to store identification data. This choice is based on: (i) quick access, (ii) it is easy to maintain copies and (iii) directory is designed to receive many more requests for reading as for writing.

Library of plans. It contains (i) a set of plans directed by the global purpose (taking care of patients) and subpurposes (care of the sick, therapy, etc.) useful for the processes of planning and negotiation. These two concepts are the subject of our forthcoming article. (ii) A set of information necessary for actors to realize their tasks. Every plan is characterized by the actions to be executed and the resources to be used. Example: nurse send an activity report to the doctor explaining the actions performed in order to determine the patient's status. The task is to give the patient's daily status (Ex. diabetic elderly).

The plan is (set of actions to be performed):

1. Measure the weight

2. Check blood pressure

3. Check body temperature

4. Check blood sugar level

5. ECG

Furthermore, to better understand the implementation of the plan, and as XML will be used for the description and interpretation of the content of the messages exchanged between the different user agents. XML perfectly illustrates the structure of the plan to execute (see section $\mathrm{G}$ ).

\section{Control agent}

It is a reactive agent. This agent must manage all associated databases in the data center to make their manipulation uniform. Also, the control agent manages data security and protection of privacy of patients and actors (confidentiality). Thus, it uses authentication and encryption mechanisms. It controls all the formulated queries to databases (radiology data, laboratory data, etc.). The solution of the control agent allows full control of UA, the voluntary or involuntary access to shared medical records which may contain sensitive data. The authentication system allows actors to prove easily their identities and get access to shared databases without threatening the security of system. Control agent has the advantage of supporting the adaptability, reuse and independence.

\section{E. Shared Databases}

At this level, this is to ensure the entire life cycle of stored medical data of patients. Each database is not only a trace of information, but also a tool for communication, information and coordination between the different structures of the hospital to taking care of patients.

The medical actors will be able to access the THC's databases via Internet with any mobile device (e.g. PDA). The patient will access his medical records using credentials from these devices. Each actor has his own credential key automatically generated and delivered by the hospital authorities to access data. So, databases are composed of:

Medical Knowledge. Relating to disease and medicine. Electronic Medical Record (EMR). The medical record is an essential tool to share information for cooperation. It consists of an important amount of information. The EMR is stored and capable to be consulted even from distance. The sharing of medical information is vital in medicine concerning at the same time: diagnosis, prognosis, analysis and care continuance. It is a progress factor for better medical care, faster and adapted, and so, systematically transmits information to other participants. To improve information traffic and functioning of our approach, the medical record is primordial, it helps to: (i) Minimize medical errors, (ii) Accelerate knowledge diffusion and (iii) Help decision making.

EMR is brought to evolve and to be updated more frequently. It allows a permanent communication between actors in an interprofessional context in order to 
PAPER

Agent And Mobile ToOls for Telehomecare in DeVeloping Countries: An ArChitecture Approach

cooperate, and to inquire mutually their conclusions' result and actions. Every actor has to find any indication and any information which is useful for understanding situations: to keep information traceability, to orchestrate, to organize interventions, to share and to exchange information to assure the coherence interventions, the continuance and the care quality. EMR guarantees the interoperability among UA in the sense of coordination to collect, enrich and exchange structured medical data.

Furthermore, a virtual collaborative working space reserved for all user actors allows them to work while being geographically distant and by keeping a permanent assistance around the patient.

\section{F. Information exchange}

In eHealth is mandatory to use a standardized communication. The development of Telehomecare is also based on standards. They have a significant contribution to interoperability in the THC. Among our contribution's objectives, are to encourage and push the evolution of normalized standards, to support the reusability and the interoperability in the field of distributed medical information. The need to transmit data in a structured form allowing automatic processing has resulted in the development of standard messages. Therefore, it is relatively easy to interconnect systems. Making them interoperable requires an agreement on the structure of exchanged data. But it is not always easy to interconnect systems. For this purpose the international standardization in the field of health plays a vital role to ensure some interoperability in medical information exchange. It should also be noted the predominant place occupied by the XML standard. XML is the standard of data description. This standard promotes the convergence of all standards and norms above [15];[16]. In our proposition, all exchanges of messages and information are based on XML.

\section{G. Inter-Agent Communication}

The success of our approach also depends on an effective communication throughout its progress. To analyze data, identify information, maintain knowledge, communicate syntheses, recommendations and decisions-making of agents that are different in their functions and activities, require an unfailing communication. So, it is necessary to assure a permanent communication. Several mechanisms of communication are possible: message exchange, methods invocation and the use of the "blackboard" mechanism. Consequently, standardized inter-agent communication languages should be supplied. In our approach, we use the ACL to formulate messages and the XML to describe the contents of messages. The use of ACL-XML in inter-agent communication permits to achieve a first level of interoperability by surpassing the problem of heterogeneous exchanges among the different actors in the hospital. The success of the information diffusion among actors resides certainly in the communication protocols standardization. The following example illustrates the use of a message in ACL FIPA containing a report structured in XML:

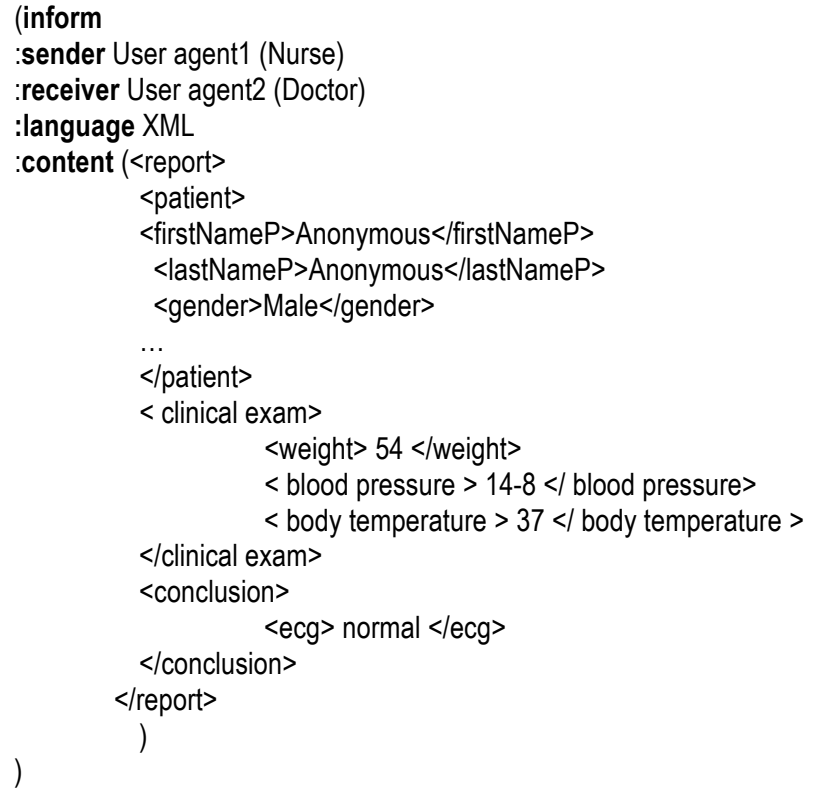

\section{MoBILE TOOLS}

\section{A. Sensors}

These devices replace the necessity of manual data gathering. The sensors collect different vital signs. The types of vital signs may include blood pressure, pulse rate, body temperature, etc. There are several practical advantages in using these devices, such as:

- It provides always-on, real-time data collecting;

- It eliminates manual collecting work and possibility of typing errors; and

- It facilitated the deployment process, as wireless networking means no need for cabling or other physical setup.

\section{B. Communication tools}

In the last few years, mobile technologies in particular emerged and become very important. Some countries reached an effective penetration of $100 \%$ such as Finland. In 2012, there are 35 million users of mobile phones in Algeria that represent $95 \%$ of the population [17]. All current phones can access wireless data networks whenever the phone has a signal, through GPRS, 3G, 4G connections or even through Wi-Fi (wireless fidelity), that enables data exchange via the Internet. Telehealth systems in the form of online and mobile tools are already opening up the possibilities for reduced hospitalization and an increased homecare [18]. Indeed, ICT tools can be used to access a wide variety of technological solutions for communication, including text messaging, gathering and monitoring data, diagnosis and treatment at distances, and retrieving electronic health records [19];[5];[ 20].

Actors of the THC are different in terms of forming, competence or task to be carried out. This variability is translated by a perception mechanism of knowledge and various access modes to information. Then, it is necessary to take into account this variety, to offer to the actors the adapted and efficient interaction modes, a better diffusion, and a data acquisition to interoperate. 
Mobile tools such as smart phone constitute a real condition for the THC success. Their uses on behalf of all the participating actors appear to be: the power to share information, knowledge and taking decisions through cooperation.

\section{PRototyPe IMPLEMENTATION}

In order to study the feasibility of the proposed architecture, we implemented a prototype using standards (see figure 3). We used XML technology to represent the information exchanged among agents via the standard communication language ACL-FIPA (the possibility of using standards like HL7 v3 message, CDA, DICOM, etc. encoded in XML [21]). Indeed, the system can interact with other applications in hospital for example through the XML exported files. The platform which gets closer most our criteria is JADE (Java Agent Development Framework) [22]. It is a platform of agents' creation that takes into account FIPA specifications for the Multi-Agent Systems (MAS) interoperability. Figure 4 shows the doctor interface through PDA.

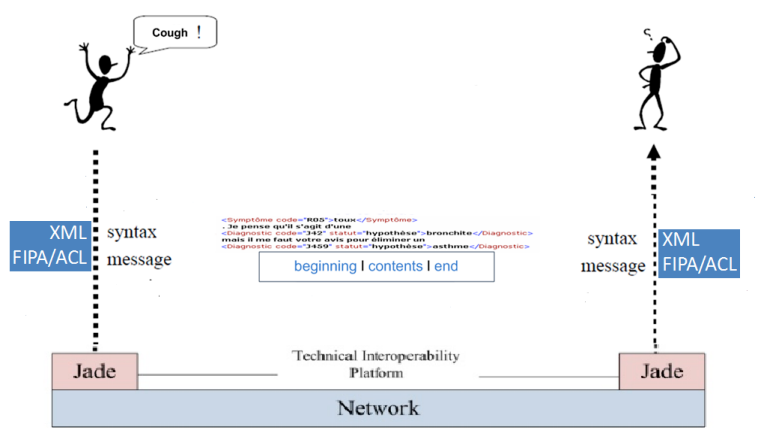

Figure 3. The overall implementation

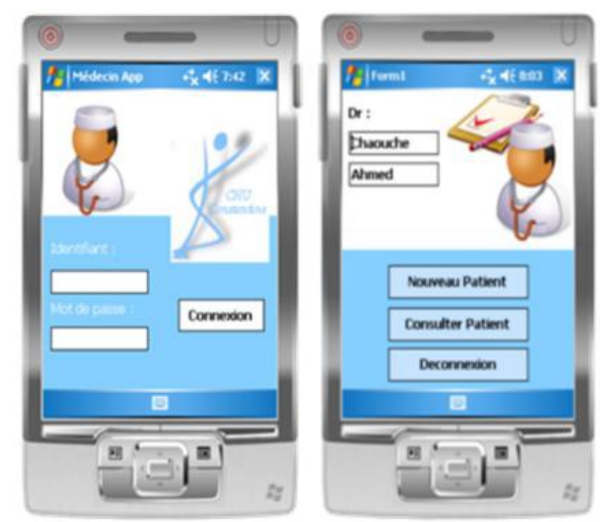

Figure 4. Doctor interface

The following Java code represents the implementation of the UserAgent class. These classes are extensions of the basic Agent class defined in JADE.

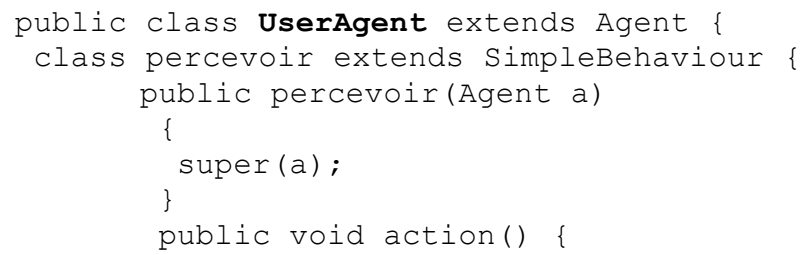

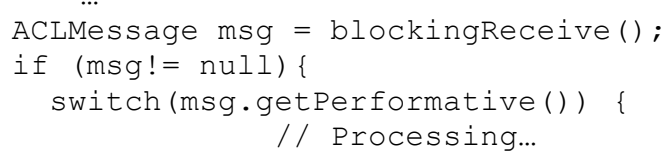

Key benefits of using this architecture:

- Real-time Services (e.g. to support remote medical consultations)

- Health actors and in particular doctors can easily access test results previously made, and information about patients.

- Avoid unnecessary interactions between health actors.

- Efficiency side, health actors spend much less time and effort to search information, which allows to organize activities and to avoid delays in care.

- Patients undergo fewer redundant tests.

Specially reduction in:

- $\quad$ home visits (lack of doctor and nurse)

- hospital stays (limited number of beds and hospitals)

Disadvantages:

- $\quad$ high cost of equipments

\section{RELATED WORK}

In recent years, many countries have tried to apply information and communication technologies to the healthcare system and especially to THC. . In the study of [23], The status of telehomecare in developing countries in Africa and other regions differs from that in other areas. The problem is accentuated by a lack of infrastructure and health personnel. In the work of [24], an information platform for tele-healthcare services was implemented. The platform deployed a service-oriented architecture, which consisted of HL7 standard messages and web service components. The prototyping system was pretested and evaluated in a homecare department of hospital and a community management center for chronic disease monitoring. In Canada the effectiveness of home tele-healthcare for diabetes is studied and proposed a decision framework to address complex issues of adopting home telehealth technology into practice [25]. In general, it has been concluded that home telehealth systems produce accurate and reliable data and capable of improving patient management [26]. The aim of [27] was to review existing studies describing the use of ICT in home care for communication between patients, family members, and healthcare professionals. The work of Tollinsky [28] described the recent incorporation of "telehomecare" services for older adults living with chronic disease in Northern Ontario communities. Kort and Hoof [29] distinguished three categories of THC technologies: (i) remote telecare, (ii) activity monitoring, and (iii) a category comprising telemedicine and e-health solutions and services. There are numerous barriers to the implementation of Dutch THC technologies projects. The studies in [30] showed that THC allows nurses to treat more patients and to deliver equivalent care. 


\section{CONCLUSION AND FUTURE WORK}

In this paper, we have presented an information system architecture supporting telehomecare. This architecture is based on agents and mobile tools. This architecture allows interoperability and easy data access. Also, it facilitates flexible interaction between doctor and nurse, patient and doctor or patient and nurse through the Internet. Software agents help to ensure the availability of data in healthcare and can be used to support processes, for example, information retrieval. Every agent assists an autonomous actor. These actors are geographically scattered belonging to diverse information's systems of hospital. In the current state we are inclined to study important aspects such as the coordination among different health actors for taking care of patients at home.

In terms of future work, it is desirable to deploy our architecture in a realistic environment to further evaluate and improve our study. Besides, the future work may produce a system with more functions and higher security mechanism in further project.

\section{REFERENCES}

[1] W. Jobe, "Native Apps vs. Mobile Web Apps", International Journal of Interactive Mobile Technologies, vol. 7, no. 4, 2013. http://dx.doi.org/10.3991/ijim.v7i4.3226

[2] G. Demiris, S.M. Speedie, and S. Finkelstein, "Change of Patients Perceptions of TeleHomeCare", Telemedicine journal and $e$ Health, vol.3, pp241-248, 2001. http://dx.doi.org/10.1089/ 153056201316970948

[3] K. Zarour, N. Zarour, and S. Khalfi, "Inter-agent Interaction in Medical Information System: Case HomeCare", Journal of Theoretical and Applied Information Technology, Vol.11, pp.130$142,2010$.

[4] S. Nourizadeh, "Un système de télésanté contextuel avec support de qualité de service pour le maintien à domicile", Published $\mathrm{PhD}$ thesis, Institut National Polytechnique Lorraine, France, 2001.

[5] S. Koch, "Home telehealth: Current state and future trends", International Journal of Medical Informatics, vol.75, pp. 565-576, 2006. http://dx.doi.org/10.1016/j.ijmedinf.2005.09.002

[6] D. Pick, Telehomecare. http://www.personal.psu.edu/dkp118/ blogs/darlene_pick_a-portfolio/2009/04/telehome-care.html Ontario telehomecare, "What is Telehomecare?", http://telehomecare.otn.ca

[7] M.E. Stachura and E. V. Khasanshina, "Telehomecare and Remote Monitoring:An Outcomes Overview", The Advanced Medical Technology Association, 2007.

[8] T. Tambo, N.H. Petersen, E.B. Pedersen and K. Bejder, "Coherent national IT infrastructure for telehomecare - a case of hypertension measurement, treatment and monitoring", Engineering and Technology, vol.47, pp.758-765, 2010.

[9] K. Bowles, A. Baugh, "Applying research evidence to optimize telehomecare, Journal Cardiovasc Nurs", vol.22, pp.5-15, 2007. http://dx.doi.org/10.1097/00005082-200701000-00002

[10] G. Demiris, Home based e-health applications, "E-Health: Current Status and Future Trends", Studies in Health Technology and Informatics, in Demiris, G. (Ed.), vol. 106, IOS Press, Amsterdam, Berlin, Oxford, Tokyo, Washington DC, 2004, pp. 15-24.

R. Elford, "An Introduction to Telehome Car", http://www.telehealth.ca/intrototelehomecare.html

[11] M. Chan, E. Campo, D. Esteve, and J-Y. Fourniols, "Smart homes. - current features and future perspectives", Maturitas, vol.64, pp. 90-97, 2009. http://dx.doi.org/10.1016/j.maturitas.2009.07.014

[12] R. Hussein and A. Khalifa, "Telemedicine in Egypt: SWOT analysis and future trends", GMS Medizinische Informatik, Biometrie und Epidemiologie, vol.8, pp.1-16, 2008.
[13] HL7 Specification. http:www.hl7.org

[14] S. Umer, M. Afzal, M. Hussain, H.F. Ahmad and K. Latif, "Design and implementation of an automation tool for HL7 RIMto-rational database mapping", In Proc. of the 10th International HL7 Interoperability Conference (IHIC), 2009.

[15] F. Lezzar, A. Zidani and A. Chorfi , "Enabling coordination within medical settings: case of a maternity ward", Applied Medical Informatics, vol.32, pp. 39-46, 2013.

[16] A. Venter, R. Burns, M. Hefford and N. Ehrenberg, "Results of a telehealth-enabled chronic care management service to support people with long-term conditions at home", Journal of Telemedicine and Telecare, vol.18, pp. 172-175, 2012. http://dx.doi.org/10.1258/jtt.2012.SFT112

[17] European Commision, What is eHealth? ICT for health. Europe's Information Society, http://ec.europa.eu/information_society/ activities/health/whatis_enealth/index_en.htm

[18] S. Koch and M. Hägglund, "Health informatics and the delivery of care to older People", Maturitas, vol.63, pp. 195-199, 2009. http://dx.doi.org/10.1016/j.maturitas.2009.03.023

[19] B. Orgun and J. Vu, "HL7 ontology and mobile agents for interoperability in heterogeneous medical information systems", Computers in Biology and Medicine, vol.36, pp. 817-836, 2006. http://dx.doi.org/10.1016/j.compbiomed.2005.04.010

[20] Jade, Java Agent DEvelopment Framework. http://sharon.cselt.it/projects/jade/

[21] I. Nakajima, Y. Zhao and H. Juzoji, "IP-ECG for Telehomecare" www.itu.int/AFRICA2001/forum/speeches/nakajima pap.doc

[22] E-W. Huang, R-S. Rung, S-F. Chiou, F-Y. Liu and D-M. Liou, "Design and Development of a Tele-Healthcare Information System Based on Web Services and HL7 Standards", Software Tools and Algorithms for Biological Systems, Advances in Experimental Medicine and Biology, Arabnia H.R. and Tran Q.-N. (eds.), Springer, 2011, pp.599-606.

[23] A. Marilynne, B. Korabek, R. Scott, "Moving Research into Practice: A Decision Framework for Integrating Home Telehealth into Chronic Illness Care", International Journal of Medical Informatics, vol.75, pp.786-794, 2006. http://dx.doi.org/10.1016/ j.ijmedinf.2006.05.041

[24] P. Guy, J. Mirou, and S. Claude, "Systematic Review of Home Telemonitoring for Chronic Diseases: The Evidence Base", Journal of the American Medical Informatics Association, vol.14, pp. 269-277, 2007. http://dx.doi.org/10.1197/jamia.M2270

[25] B. Lindberg, C. Nilsson, D. Zotterman, S. Söderberg and L. Skär, "Using Information and Communication Technology in Home Care for Communication between Patients, Family Members, and Healthcare Professionals: A Systematic Review", International Journal of Telemedicine and Applications, pp.1-32, 2013. http://dx.doi.org/10.1155/2013/461829

[26] M. Tollinsky, "Telehomecare programs rolling out across the north", Northern Ontario Medical Journal, 2012.

[27] H.S.M. Kort and J. Hoof, "Telehomecare in the Netherlands : barriers to implementation", International Journal of Ambient Computing and Intelligence, vol.4, pp. 64-73, 2012. http://dx.doi.org/10.4018/jaci.2012040105

[28] The Montreal Economic Institute (MEI), “Appendix. Comparison of studies on telehomecare", 2010.

\section{AUTHOR}

K. Zarour is Associate Professor in the Department of Software Technologies and Information Systems at the University Constantine2, Constantine, Algeria. He received his $\mathrm{PhD}$ degree in computer science from the University Mentouri of Constantine, Algeria. His research interests include advanced information systems, particularly medical information systems.

This research is partially supported by the CNEPRU project under the number B*00920100175. Supported 05 November 2014. Published as resubmitted by the authors 23 March 2015. 